\title{
The Dynamic Nash Bargaining Solution for 2-Stage Cost Sharing Game ${ }^{\star}$
}

\author{
Li Yin \\ St.Petersburg State University, \\ Faculty of Applied Mathematics and Control Processes, \\ Universitetskii prospekt 35, St.Petersburg, 198504, Russia \\ E-mail: liyinrus@outlook.com \\ Home page: http://www.liyin.site
}

\begin{abstract}
The problem of constructing the Dynamic Nash Bargaining Solution in a 2-stage game is studied. In each stage, a minimum cost spanning tree game is played, all players select strategy profiles to construct graphs in the stage game. At the second stage, players may change the graph using strategy profiles with transition probabilities, which decided by players in the first stage. The players' cooperative behavior is considered. As solution the Dynamic Nash Bargaining Solution is proposed. A theorem is proved to allow the Dynamic Nash Bargaining Solution to be time-consistent.
\end{abstract}

Keywords: Dynamic Nash Bargaining, dynamic game, minimum cost spanning tree.

\section{The Model}

In the paper, we consider a 2 -stage game with spanning tree.

$* H=(Z, F)$ is a finite game tree with the initial vertex $z_{1}$.

$* Z$ is a set of vertexes in the game tree.

* $F\left(z_{1}\right)$ is a point-to-set mapping: $F\left(z_{1}\right) \subset Z$.

* In the second stage, $F\left(z_{1}\right)$ is the set of vertexes on the tree-like graph.

*i.e. $F\left(z_{1}\right)=Z \backslash\left\{z_{1}\right\}$.

* $m\left(z_{1}\right)$ is the number of elements in the set $F\left(z_{1}\right)$.

* $\Gamma\left(z_{1}\right)$ is the game starting from initial vertex $z_{1}$.

* Similarly, $\Gamma\left(z_{k}\right)$ is the subgame starting from the vertex $z_{k} \in F\left(z_{1}\right)$.

* $N=\{1,2, \ldots, n\}$ is a finite set of players.

$* N^{\prime}=N \cup\{0\} .\{0\}$ is the source.

* $G\left(N^{\prime}, E\right)=\left\{(i, j): \forall i, j \in N^{\prime}\right\}$ is a graph over $N^{\prime}$.

$* E$ is the set of all edges.

* If $\exists\left(i_{1}, i_{2}\right),\left(i_{2}, i_{3}\right), \ldots,\left(i_{n-1}, i_{n}\right)$, such that $\left(i_{k}, i_{k+1}\right) \in G\left(N^{\prime}, E\right), 1 \leq k \leq n-1$, and $i_{1}=i, i_{n}=j$, then two vertexes $i$ and $j \in N^{\prime}$ are said to be connected in $G$.

* If all $i, j$ are connected in $G$, a graph $G$ is called connected over $N^{\prime}, \forall i, j \in N^{\prime}$.

* $\mathcal{G}_{N^{\prime}}$ is the set of connected graphs over $N^{\prime}$.

Definition 1. The cost of connections is represented by a cost matrix ( $\mathrm{Li}, 2016)$

$$
C_{m}=\left(c_{i j}\right)_{(n+1) \times(n+1)},
$$

where $c_{i j}=c_{j i}>0$ is the cost of connecting $i$ and $j, i \neq j \in N^{\prime}$. In the paper, $c_{i 0}=c_{0 i}$ is a nonnegative constant, and cost matrices are nonnegative, symmetric.

\footnotetext{
^ This work was supported by the China Scholarship Council (No.201508090030).
} 
Definition 2. At each stage, player $i$ chooses a vector

$$
x_{i}=\left(x_{i, 1}, \ldots, x_{i, i-1}, x_{i, i+1}, \ldots, x_{i, n}\right),
$$

where $x_{i, j} \in X_{i, j}$ is a strategy of player $i$ against player $j$. Similarly, $x_{j, i} \in X_{j, i}$ is a strategy of player $j$ against player $i$. At different stages, the set $X_{i, j}$ may be different for the player $i, \forall i, j \in N$

Definition 3. At each stage, the cost of edge $(i, j)$ is defined as

$$
c_{i j}=c_{j i}=f_{c}\left(x_{i, j}, x_{j, i}\right), c_{i 0}=c_{0 i}>0, \forall i, j \in N .
$$

where function $f_{c}$ is a mapping from strategies of players $i, j$ to the set $\mathbb{R}^{+} \cup\{+\infty\}$ - the set of all possible cost of edge $(i, j)$.

Definition 4. $T_{x}\left(N^{\prime}, C_{m}\right)$ is the minimum cost spanning tree (m.c.s.t.) (Bird, 1976) over $N^{\prime}$

$$
T_{x}\left(N^{\prime}, C_{m}\right)=\arg \min _{G \in \mathcal{G}_{N^{\prime}}} \sum_{(i, j) \in G\left(N^{\prime}, E\right)} c_{i j}
$$

where $C_{m}=\left(c_{i j}\right)_{(n+1) \times(n+1)}$ is the cost matrix.

Definition 5. $C\left[T_{x}\left(N^{\prime}, C_{m}\right)\right]$ is the total cost of edges in the m.c.s.t. (Bird, 1976) $T_{x}\left(N^{\prime}, C_{m}\right)$

$$
C\left[T_{x}\left(N^{\prime}, C_{m}\right)\right]=\sum_{(i, j) \in T_{x}\left(N^{\prime}, C_{m}\right)} c_{i j}
$$

\section{Description of the Game}

\subsection{Stage 1}

Players simultaneously choose their behaviors, i.e. $n$-dimensional strategy profiles

$$
\begin{aligned}
& x^{1}\left(z_{1}\right)=\left(x_{1}^{1}\left(z_{1}\right), \ldots, x_{n}^{1}\left(z_{1}\right)\right), \\
& x_{i}^{1}\left(z_{1}\right)=\left(x_{i, 1}^{1}\left(z_{1}\right), \ldots, x_{i, i-1}^{1}\left(z_{1}\right), x_{i, i+1}^{1}\left(z_{1}\right), \ldots, x_{i, n}^{1}\left(z_{1}\right)\right),
\end{aligned}
$$

where $x_{i, j}^{1}\left(z_{1}\right) \in X_{i, j}^{1}$ is a strategy of player $i$ against player $j, \forall i \neq j, i, j \in$ $N$. By definition (1)(2), this means that at stage 1 player $i$ and player $j$ choose their strategies $x_{i, j}^{1}\left(z_{1}\right)$ and $x_{j, i}^{1}\left(z_{1}\right)$ and build an edge $(i, j)$, and $c_{i j}^{1}=c_{j i}^{1}=$ $f_{c}\left(x_{i, j}^{1}\left(z_{1}\right), x_{j, i}^{1}\left(z_{1}\right)\right)$.

\subsection{Stage 2}

The game proceeds to the second stage with probability, which depends on strategies of players chosen on stage 1 . The transition probabilities are defined as following

$$
\begin{aligned}
p\left(z_{1}, z_{k}, x_{1}^{1}\left(z_{1}\right), \ldots, x_{n}^{1}\left(z_{1}\right)\right) & =p\left(z_{1}, z_{k}, x^{1}\left(z_{1}\right)\right) \geq 0, \\
\sum_{z_{k} \in F\left(z_{1}\right)} p\left(z_{1}, z_{k}, x^{1}\left(z_{1}\right)\right) & =1
\end{aligned}
$$

where $p\left(z_{1}, z_{k}, x^{1}\left(z_{1}\right)\right)$ is the probability that the game moves from initial vertex $z_{1}$ to the vertex $z_{k}$.

Assume that each vertex $z_{k} \in F\left(z_{1}\right)$ is associated with a matrix called $\alpha$-matrix. 
Definition 6. The $\alpha$-matrix of the stage game in vertex $z_{k}$ is defined as

$$
\begin{aligned}
\alpha\left[p\left(z_{1}, z_{k}, x^{1}\left(z_{1}\right)\right)\right] & =\left(\begin{array}{cccccc}
\infty & 1 & 1 & \ldots & 1 & 1 \\
1 & \infty & \alpha_{1,2} & \ldots & \alpha_{1, n-1} & \alpha_{1, n} \\
1 & \alpha_{2,1} & \ldots & \ldots & \ldots & \ldots \\
\ldots & \ldots & \ldots & \ldots & \ldots & \ldots \\
1 & \alpha_{n-1,1} & \ldots & \ldots & \infty & \alpha_{n-1, n} \\
1 & \alpha_{n, 1} & \ldots & \ldots & \alpha_{n, n-1} & \infty
\end{array}\right), z_{k} \in F\left(z_{1}\right) \\
\alpha_{i, j} & =\alpha_{j, i}=1 \text { or }+\infty, \forall i \neq j \in\{1, \ldots, n\}
\end{aligned}
$$

where $x^{1}\left(z_{1}\right)$ are the strategies of players in the previous stage game starting from initial vertex $z_{1}$.

Definition 7. For two matrices $A$ and $B$ with the same dimension $(m \times n)$, the Hadamard product $A \circ B$ (Horn, 2012) is a matrix which elements are given by

$$
A \circ B=\left(\begin{array}{ccc}
a_{11} & \ldots & a_{1 n} \\
\ldots & & \ldots \\
a_{m 1} & \ldots & a_{m n}
\end{array}\right) \circ\left(\begin{array}{ccc}
b_{11} & \ldots & b_{1 n} \\
\ldots & & \ldots \\
b_{m 1} & \ldots & b_{m n}
\end{array}\right)=\left(\begin{array}{cccc}
a_{11} \times b_{11} & \ldots & a_{1 n} \times b_{1 n} \\
\ldots & & \ldots \\
a_{m 1} \times b_{m 1} & \ldots & a_{m n} \times b_{m n}
\end{array}\right)
$$

The cost matrix of the stage game in the vertex $z_{k} \in F\left(z_{1}\right)$ is defined as follows

$$
\begin{array}{r}
C_{m}\left(z_{k}\right)=\alpha\left[p\left(z_{1}, z_{k}, x^{1}\left(z_{1}\right)\right)\right] \circ\left\{c_{i j}^{2}\right\}_{(n+1) \times(n+1)}, \\
c_{i j}^{2}=c_{j i}^{2}=f_{c}\left(x_{i, j}^{2}\left(z_{k}\right), x_{j, i}^{2}\left(z_{k}\right)\right), c_{i 0}=c_{0 i}>0
\end{array}
$$

where $x_{i, j}^{2}\left(z_{k}\right) \in X_{i, j}^{2}$ is a strategy of player $i$ against player $j, \forall i \neq j, i, j \in N$, $z_{k} \in F\left(z_{1}\right)$.

Example 1. The Fig. 1 shows, how the strategy profiles $x^{1}\left(z_{1}\right)$ can influence the game played in the second stage.

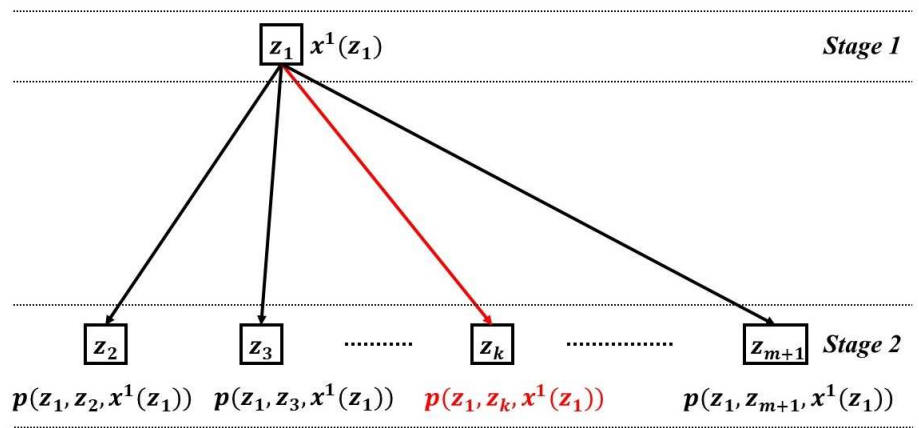

Fig. 1. The diagram of the 2-stage game with spanning tree

\section{Cooperative Game}

In 2-stage m.c.s.t. game assume that the total cost of players is the sum of the cost of players on both stages (Parilina, 2015). 
During the game suppose that the path $z^{\prime}, z^{\prime \prime}$ is realized. Let $x_{i}\left(z^{\prime}\right)=x_{i}^{1}\left(z_{1}\right)$ and $x_{i}\left(z^{\prime \prime}\right)=\left(x_{i}^{2}\left(z_{2}\right), \ldots, x_{i}^{2}\left(z_{k}\right)\right), i \in N, z_{k} \in F\left(z_{1}\right)$. The cooperative solution of the game $\Gamma\left(z_{1}\right)$ for the set $N^{\prime}$ at the first stage is defined as follows:

$$
\begin{array}{r}
V^{1}\left(N^{\prime}, z_{1}\right) \\
=\min _{x(\cdot)}\left\{C\left[T_{x^{1}\left(z_{1}\right)}\left(N^{\prime}, C_{m}\left(z_{1}\right)\right)\right]+\sum_{z_{k} \in F\left(z_{1}\right)} p\left(z_{1}, z_{k}, x^{1}\left(z_{1}\right)\right) C\left[T_{x^{2}\left(z_{k}\right)}\left(N^{\prime}, C_{m}\left(z_{k}\right)\right)\right]\right\} \\
=C\left[T_{\bar{x}^{1}\left(z_{1}\right)}\left(N^{\prime}, C_{m}\left(z_{1}\right)\right)\right]+\sum_{z_{k} \in F\left(z_{1}\right)} p\left(z_{1}, z_{k}, \bar{x}^{1}\left(z_{1}\right)\right) C\left[T_{\bar{x}^{2}\left(z_{k}\right)}\left(N^{\prime}, C_{m}\left(z_{k}\right)\right)\right], \\
V^{1}\left(\emptyset, z_{1}\right)=0, z_{k} \in F\left(z_{1}\right),
\end{array}
$$

where $p\left(z_{1}, z_{k}, \bar{x}^{1}\left(z_{1}\right)\right), k \in\left\{k: z_{k} \in F\left(z_{1}\right)\right\}$ are defined in $(5), V^{1}\left(N^{\prime}, z_{1}\right)$ is the value of characteristic function for set $N^{\prime}$ in the game $\Gamma\left(z_{1}\right)$.

Strategies $\bar{x}_{i}(\cdot), i \in N$ are called cooperative strategies, and strategy profile $\bar{x}(\cdot)=\left(\bar{x}_{i}(\cdot), i \in N\right)$ - cooperative strategy profile.

\subsection{The Value of the Game for the Player in the Game $\Gamma\left(z_{1}\right)$}

The value of the game for the player $i \in N$ is defined as the value of the zero-sum game in which player $i$ plays against players from $N \backslash\{i\}$. In the zero-sum game, all players in $N \backslash\{i\}$ don't want to be connected to the source. Thus in this situation assuming that players in $N \backslash\{i\}$ are out and the m.c.s.t. contains only one edge $(i, 0), i \in N$, which means that the cost of this unique edge in each stage game is the cost of m.c.s.t. of this stage.

$$
\begin{gathered}
V^{1}\left(\{i\}^{\prime}, z_{1}\right)= \\
\min _{x(\cdot)}\left\{C\left[T_{x^{1}\left(z_{1}\right)}\left(\{i\}^{\prime}, C_{m}^{\{i\}^{\prime}}\left(z_{1}\right)\right)\right]+\sum_{z_{k} \in F\left(z_{1}\right)} p\left(z_{1}, z_{k}, x^{1}\left(z_{1}\right)\right) C\left[T_{x^{2}\left(z_{k}\right)}\left(\{i\}^{\prime}, C_{m}^{\{i\}^{\prime}}\left(z_{k}\right)\right)\right]\right\} \\
=C\left[T_{\bar{x}^{1}\left(z_{1}\right)}\left(\{i\}^{\prime}, C_{m}^{\{i\}^{\prime}}\left(z_{1}\right)\right)\right]+\sum_{z_{k} \in F\left(z_{1}\right)} p\left(z_{1}, z_{k}, \bar{x}^{1}\left(z_{1}\right)\right) C\left[T_{\bar{x}^{2}\left(z_{k}\right)}\left(\{i\}^{\prime}, C_{m}^{\{i\}^{\prime}}\left(z_{k}\right)\right)\right] \\
=c_{i 0}+\sum_{z_{k} \in F\left(z_{1}\right)} p\left(z_{1}, z_{k}, \bar{x}^{1}\left(z_{1}\right)\right) c_{i 0}=2 c_{i 0}
\end{gathered}
$$

where $p\left(z_{1}, z_{k}, \bar{x}^{1}\left(z_{1}\right)\right), k \in\left\{k: z_{k} \in F\left(z_{1}\right)\right\}$ are defined in $(5) . C^{\{i\}^{\prime}}\left(z_{1}\right)$ and $C^{\{i\}^{\prime}}\left(z_{k}\right)$ is the cost matrix restricted to $\{i\}^{\prime}$ and is determined by (1) and (7). It means that $C^{\{i\}^{\prime}}\left(z_{1}\right)$ and $C^{\{i\}^{\prime}}\left(z_{k}\right)$ are sub-matrices of matrix $C\left(z_{k}\right)$.

\subsection{The Value of the Game for the Player in the Game $\Gamma\left(z_{k}\right)$}

Suppose that the subgame $\Gamma\left(z_{k}\right)$ happened in the vertex $z_{k} \in F\left(z_{1}\right)$ of the tree-like graph $H=(Z, F)$.

According to the definition (5), $p\left(z_{1}, z_{k}, x^{1}\left(z_{1}\right)\right)$ - the transition probability to proceed from initial vertex $z_{1}$ to the vertex $z_{k}$. Thus, the cooperative solution of the subgame $\Gamma\left(z_{k}\right)$ for the set $N^{\prime}$ at the second stage is defined as follows:

$$
\begin{gathered}
V^{2}\left(N^{\prime}, z_{k}\right)=\min _{x^{2}(\cdot)} C\left[T_{x^{2}\left(z_{k}\right)}\left(N^{\prime}, C_{m}\left(z_{k}\right)\right)\right]=C\left[T_{\bar{x}^{2}\left(z_{k}\right)}\left(N^{\prime}, C_{m}\left(z_{k}\right)\right)\right], \\
V^{2}\left(\emptyset, z_{k}\right)=0,
\end{gathered}
$$


where $C\left(z_{k}\right)$ is defined by $(7)$. Strategies $\bar{x}_{i}^{2}(\cdot), i \in N$ are a cooperative strategies. Strategy profile $\bar{x}^{2}(\cdot)=\left(\bar{x}_{i}^{2}(\cdot), i \in N\right)$ is a cooperative strategy profile. $V^{2}\left(N^{\prime}, z_{k}\right)$ is the value of characteristic function for set $N^{\prime}$ in the subgame $\Gamma\left(z_{k}\right)$.

In a similar way, the value of the game for the player $i \in N$ is defined as following

$$
\begin{aligned}
V^{2}\left(\{i\}^{\prime}, z_{k}\right) & =\min _{x^{2}(\cdot)} C\left[T_{x^{2}\left(z_{k}\right)}\left(\{i\}^{\prime}, C_{m}^{\{i\}^{\prime}}\left(z_{k}\right)\right)\right] \\
& =C\left[T_{\bar{x}^{2}\left(z_{k}\right)}\left(\{i\}^{\prime}, C_{m}^{\{i\}^{\prime}}\left(z_{k}\right)\right)\right]=c_{i 0},
\end{aligned}
$$

where the subgame in the vertex $z_{k}$ with the probability $p\left(z_{1}, z_{k}, \bar{x}^{1}\left(z_{1}\right)\right), z_{k} \in F\left(z_{1}\right)$ which are defined in (5), $C^{\{i\}^{\prime}}\left(z_{k}\right)$ is the cost matrix restricted to $\{i\}^{\prime}$.

\section{The Dynamic Nash Bargaining Solution}

Let $\left(H_{1}\left(z_{1}\right), \ldots, H_{n}\left(z_{1}\right)\right) \in S_{1}$ be cost vector in the game $\Gamma\left(z_{1}\right)$, the set of all possible costs is defined as

$$
S_{1}=\left\{H_{i}\left(z_{1}\right): H_{i}\left(z_{1}\right) \geq 0, i \in N\right\}
$$

and the value of the game for each player is $V^{1}\left(\{i\}^{\prime}, z_{1}\right)=2 c_{i 0}, i \in N$. Here, $S_{1}$ is bargaining set, and $V^{1}\left(\{1\}, z_{1}\right), \ldots, V^{1}\left(\{n\}, z_{1}\right) \in S_{1}$ - disagreement point.

Consider the following expression:

$$
\begin{gathered}
\max _{H_{i}\left(z_{1}\right) \leq V^{1}\left(\{i\}^{\prime}, z_{1}\right),\left(H_{1}\left(z_{1}\right), \ldots, H_{n}\left(z_{1}\right)\right) \in S_{1}} \prod_{i=1}^{n}\left[V^{1}\left(\{i\}^{\prime}, z_{1}\right)-H_{i}\left(z_{1}\right)\right] \\
=\prod_{i=1}^{n}\left[V^{1}\left(\{i\}^{\prime}, z_{1}\right)-\bar{H}_{i}\left(z_{1}\right)\right], i \in N .
\end{gathered}
$$

Vector $\left(\bar{H}_{1}\left(z_{1}\right), \ldots, \bar{H}_{n}\left(z_{1}\right)\right)$ is called Nash bargaining solution.

At the second stage, if the game proceeds to the stage game on the vertex $z_{k} \in F\left(z_{1}\right)$ with probability $p\left(z_{1}, z_{k}, \bar{x}^{1}\left(z_{1}\right)\right)$, the set of all possible costs is defined as

$$
S_{k}=\left\{H_{i}\left(z_{k}\right): H_{i}\left(z_{k}\right) \geq 0, i \in N .\right\}
$$

and, the Nash bargaining solution in one stage m.c.s.t. game is defined as follows:

$$
\begin{gathered}
\max _{H_{i}\left(z_{k}\right) \leq V^{2}\left(\{i\}^{\prime}, z_{k}\right),\left(H_{1}\left(z_{1}\right), \ldots, H_{n}\left(z_{1}\right)\right) \in S_{k}} \prod_{i=1}^{n}\left[V^{2}\left(\{i\}^{\prime}, z_{k}\right)-H_{i}\left(z_{k}\right)\right] \\
=\prod_{i=1}^{n}\left[V^{2}\left(\{i\}^{\prime}, z_{k}\right)-\bar{H}_{i}\left(z_{k}\right)\right], i \in N .
\end{gathered}
$$

Time consistency of the cooperative solution concept was introduced for the first time in (Petrosyan, 2006).

Using the IDP (Imputation Distribution Procedure) the Dynamic Nash Bargaining Solution is constructed (Junnan, 2018).

Definition 8. Imputation distribution procedure of the Nash Bargaining Solution in 2 -stage m.c.s.t. game is a scheme $\beta=\left(\beta^{1}, \beta^{2}\right)$ s.t.

$$
\beta_{i}^{1}=\bar{H}_{i}\left(z_{1}\right)-\sum_{z_{k} \in F\left(z_{1}\right)} p\left(z_{1}, z_{k}, \bar{x}^{1}\left(z_{1}\right)\right) \bar{H}_{i}\left(z_{k}\right), \forall i \in N
$$



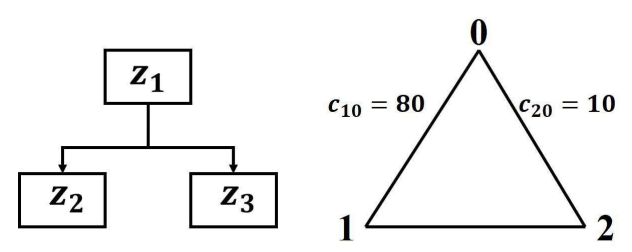

Fig. 2. The figure on the left side is the tree-like graph of the game. The figure on the right side is the graph at each stage game.

$$
\beta_{i}^{2}=\sum_{z_{k} \in F\left(z_{1}\right)} p\left(z_{1}, z_{k}, \bar{x}^{1}\left(z_{1}\right)\right) \bar{H}_{i}\left(z_{k}\right), \forall i \in N
$$

where $p\left(z_{1}, z_{k}, \bar{x}^{1}\left(z_{1}\right)\right)$ is the transition probability from the initial stage to the stage game $\Gamma\left(z_{k}\right), z_{k} \in F\left(z_{1}\right)$.

Definition 9. The Nash Bargaining Solution $H_{i}\left(z_{1}\right), i \in N$ is called time consistent in the game with spanning tree, if there exists a nonnegative IDP $\left(\beta_{i}^{1} \geq 0, \beta_{i}^{2} \geq\right.$ $0, \forall i \in N)$ such that the following condition holds:

$$
\begin{gathered}
\bar{H}_{i}\left(z_{1}\right)=\beta_{i}^{1}+\sum_{z_{k} \in F\left(z_{1}\right)} p\left(z_{1}, z_{k}, \bar{x}^{1}\left(z_{1}\right)\right) \bar{H}_{i}\left(z_{k}\right), \forall i \in N, \\
\sum_{z_{k} \in F\left(z_{1}\right)} p\left(z_{1}, z_{k}, \bar{x}^{1}\left(z_{1}\right)\right) \bar{H}_{i}\left(z_{k}\right)=\beta^{2}, \forall i \in N,
\end{gathered}
$$

where $p\left(z_{1}, z_{k}, \bar{x}^{1}\left(z_{1}\right)\right)$ is the transition probability from the initial stage to the stage game $\Gamma\left(z_{k}\right), z_{k} \in F\left(z_{1}\right)$.

Unfortunately, in 2-stage m.c.s.t. games the IDP $\beta$ may take negative value.

Proposition 1. Constructed above IDP $\beta$ for the Dynamic Nash Bargaining Solution $\left(\bar{H}_{1}\left(z_{1}\right), \ldots, \bar{H}_{n}\left(z_{1}\right)\right)$ is time inconsistent.

We propose a counterexample in order to verify the proposition.

Example 2. In this example, we consider a two-person game with spanning tree as an illustration in Fig.2.

The set of players is $N=\{1,2\}$, and the source is $\{0\}, N^{\prime}=N \cup\{0\}$. The sets of strategies, which player 1 uses against player 2 , are $X_{1,2}^{1}=\{3,4\}, X_{1,2}^{2}=\{6,7\}$, and the sets of strategy, which player 2 uses against player 1 , are $X_{2,1}^{1}=\{6,2\}$, $X_{2,1}^{2}=\{8,3\}$. Assume that there are two vertexes $z_{2}, z_{3}$ following after the initial vertex $z_{1}$ in the game. The tree-like graph as shown in Fig.2 on the left side.

As shown in Fig.2, in each stage there is a graph over $N^{\prime}$. Assume that edges $(0,1),(0,2)$ are fixed and the cost of edges are $c_{01}=c_{10}=80, c_{02}=c_{20}=10$. The function $f_{c}$ is defined as $f_{c}=x_{1,2} \times x_{2,1}, x_{1,2} \in X_{1,2}, x_{2,1} \in X_{2,1}$.

The matrices of the stage game in vertexes $z_{2}$ and $z_{3}$ are described as follows

$$
\alpha\left[p\left(z_{1}, z_{2}, x^{1}\left(z_{1}\right)\right)\right]=\left(\begin{array}{ccc}
\infty & 1 & 1 \\
1 & \infty & 1 \\
1 & 1 & \infty
\end{array}\right), \alpha\left[p\left(z_{1}, z_{3}, x^{1}\left(z_{1}\right)\right)\right]=\left(\begin{array}{ccc}
\infty & 1 & 1 \\
1 & \infty & \infty \\
1 & \infty & \infty
\end{array}\right)
$$


where $x_{i}^{1}\left(z_{1}\right) \in X_{i}^{1}$ is the strategy profile of players in the first stage, $i \in N$.

In the case of different strategy profiles of players, the game's transition probabilities are

$$
\begin{gathered}
x^{1}\left(z_{1}\right)=(3,6): p\left(z_{1}, z_{2}, x^{1}\left(z_{1}\right)\right)=0.5, p\left(z_{1}, z_{3}, x^{1}\left(z_{1}\right)\right)=0.5, \\
x^{1}\left(z_{1}\right)=(3,7): p\left(z_{1}, z_{2}, x^{1}\left(z_{1}\right)\right)=0.7, p\left(z_{1}, z_{3}, x^{1}\left(z_{1}\right)\right)=0.3, \\
x^{1}\left(z_{1}\right)=(4,6): p\left(z_{1}, z_{2}, x^{1}\left(z_{1}\right)\right)=0.9, p\left(z_{1}, z_{3}, x^{1}\left(z_{1}\right)\right)=0.1, \\
x^{1}\left(z_{1}\right)=(4,7): p\left(z_{1}, z_{2}, x^{1}\left(z_{1}\right)\right)=0.15, p\left(z_{1}, z_{3}, x^{1}\left(z_{1}\right)\right)=0.85 .
\end{gathered}
$$

According to above-mentioned analysis, in 2-stage game we get the value of the game for each player in the game $\Gamma\left(z_{1}\right)$ :

$$
V^{1}\left(\{1\}^{\prime}, z_{1}\right)=160, V^{1}\left(\{2\}^{\prime}, z_{1}\right)=20
$$

The value of the game for each player in the the subgame $\Gamma\left(z_{2}\right)$ :

$$
V^{2}\left(\{1\}^{\prime}, z_{2}\right)=80, V^{1}\left(\{2\}^{\prime}, z_{2}\right)=10
$$

The value of the game for each player in the the subgame $\Gamma\left(z_{3}\right)$ :

$$
V^{2}\left(\{1\}^{\prime}, z_{3}\right)=80, V^{1}\left(\{2\}^{\prime}, z_{3}\right)=10
$$

The value of characteristic function for set $N^{\prime}$ in the game $\Gamma\left(z_{1}\right)$ :

$$
V^{1}\left(N^{\prime}, z_{1}\right)=57.4
$$

The Nash bargaining solution in the game $\Gamma\left(z_{1}\right)$,

$$
\bar{H}_{1}\left(z_{1}\right)=57.4, \bar{H}_{2}\left(z_{1}\right)=0
$$

The Nash bargaining solution in the game $\Gamma\left(z_{2}\right)$,

$$
\bar{H}_{1}\left(z_{2}\right)=16, \bar{H}_{2}\left(z_{2}\right)=0
$$

The Nash bargaining solution in the game $\Gamma\left(z_{3}\right)$,

$$
\begin{gathered}
\bar{H}_{1}\left(z_{3}\right)=80, \bar{H}_{2}\left(z_{3}\right)=10 \\
\bar{x}^{1}\left(z_{1}\right)=\left(\bar{x}_{1}^{1}\left(z_{1}\right), \bar{x}_{2}^{1}\left(z_{1}\right)\right), \bar{x}_{1}^{1}\left(z_{1}\right)=4, \bar{x}_{2}^{1}\left(z_{1}\right)=6 \\
p\left(z_{1}, z_{2}, \bar{x}^{1}\left(z_{1}\right)\right)=0.9, p\left(z_{1}, z_{3}, \bar{x}^{1}\left(z_{1}\right)\right)=0.1
\end{gathered}
$$

We construct IDP $\beta$ for the Dynamic Nash Bargaining Solution,

$$
\begin{gathered}
\beta_{2}^{1}=\bar{H}_{2}\left(z_{1}\right)-p\left(z_{1}, z_{2}\right) \bar{H}_{2}\left(z_{1}\right)-p\left(z_{1}, z_{3}\right) \bar{H}_{2}\left(z_{3}\right) \\
=0-0.9 \times 0-0.1 \times 10=-1 \leq 0
\end{gathered}
$$

The Dynamic Nash Bargaining Solution in the example is time inconsistent. 


\section{Results}

Theorem 1. If in a 2-stage game with spanning tree $\Gamma\left(z_{1}\right)$, the following conditions hold

$$
\bar{H}_{i}\left(z_{1}\right) \geq \bar{H}_{i}\left(z_{k}\right), \forall i \in N
$$

then, the Dynamic Nash Bargaining Solution $\left(\bar{H}_{1}\left(z_{1}\right), \ldots, \bar{H}_{n}\left(z_{1}\right)\right)$ is time consistent.

Proof. The proof follows immediately from the definition of $\beta^{1}$ and $\beta^{2}$.

Multiply both side of $(15)$ on $p\left(z_{1}, z_{k}, \bar{x}\left(z_{1}\right)\right) \geq 0$ and taking in account that

$$
\sum_{z_{k} \in F\left(z_{1}\right)} p\left(z_{1}, z_{k}, \bar{x}^{1}\left(z_{1}\right)\right)=1
$$

We obtain

$$
\begin{gathered}
p\left(z_{1}, z_{k}, \bar{x}\left(z_{1}\right)\right) \bar{H}_{i}\left(z_{1}\right) \geq p\left(z_{1}, z_{k}, \bar{x}\left(z_{1}\right)\right) \bar{H}_{i}\left(z_{k}\right), \\
z_{k} \in F\left(z_{1}\right), \forall i \in N
\end{gathered}
$$

thus

$$
\begin{gathered}
\sum_{z_{k} \in F\left(z_{1}\right)} p\left(z_{1}, z_{k}, \bar{x}\left(z_{1}\right)\right) \bar{H}_{i}\left(z_{1}\right) \geq \sum_{z_{k} \in F\left(z_{1}\right)} p\left(z_{1}, z_{k}, \bar{x}\left(z_{1}\right)\right) \bar{H}_{i}\left(z_{k}\right), \\
z_{k} \in F\left(z_{1}\right), \forall i \in N
\end{gathered}
$$

then

$$
\bar{H}_{i}\left(z_{1}\right) \geq \beta_{i}^{2} \geq 0, \forall i \in N
$$

and

$$
\beta_{i}^{1}=\bar{H}_{i}\left(z_{1}\right)-\beta_{i}^{2} \geq 0, \forall i \in N
$$

the theorem is proved

\section{References}

Li, Yin (2016). The dynamic Shapley Value in the game with spanning tree. Stability and Oscillations of Nonlinear Control Systems (Pyatnitskiy's Conference). 2016 International Conference. IEEE. pp. 1-4.

Bird, C.G. (1976). On cost allocation for a spanning tree: a game theoretic approach. Networks, 6(4), 335-350.

Horn, R. A., Johnson, C. R. (2012). Matrix analysis. Cambridge university press.

Petrosyan, L. (2006). Cooperative stochastic games. Advances in dynamic games, Birkhauser Boston, 139-145.

Junnan, J. (2018). Dynamic Nash Bargaining Solution for two-stage network games. Contributions to Game Theory and Management, 11(0), 66-72.

Parilina, E. M. Stable cooperation in stochastic games. Autom Remote Control, 76, 11111122 .

Granot, D., Huberman, G. (1981). Minimum cost spanning tree games. Mathematical programming, 21(1), 1-18.

Petrosyan, L. A. (1977). Stability of the Solutions of Differential Games with Several Players. Vestnik of the Leningrad State University, 19, 46-52. 